\title{
Subjective Sleep Quality in Women with Premenstrual Syndrome and its Correlation with Serum Magnesium Level
}

\author{
R. Abiramasundari ${ }^{1}$, R. Shanthini ${ }^{2}$, V. Santhosini ${ }^{3}$ \\ ${ }^{1}$ Assistant Professor, ${ }^{2}$ Associate Professor, ${ }^{3}$ Post Graduate, Department of Physiology, \\ Government Stanley Medical College and Hospital, Chennai
}

\begin{abstract}
Background: Premenstrual Syndrome (PMS) is defined as a cyclical disorder in the late luteal phase with occurrence of non-specific somatic, psychological or behavioural changes. Sleep disturbances are more common in Premenstrual syndrome. Apart from hormonal causes, micronutrients like magnesium, calcium and zinc are also involved in the pathogenesis of Premenstrual syndrome.
\end{abstract}

Objective: To assess the Subjective sleep quality in women with Premenstrual Syndrome and its correlation with serum Magnesium level.

Method: It is a cross- sectional study involving 175 women of age group 18-40 years. After obtaining ethical committee clearance, Premenstrual Syndrome is diagnosed using Premenstrual syndrome scale (PMSS). Subjective sleep quality in them are assessed using Pittsburgh Sleep Quality Index (PSQI) and then $5 \mathrm{ml}$ of venous blood are collected for Serum Magnesium Level estimation.

Results: Among the study population of 175 women with PMS, good sleep quality was reported by 87 women $(49.71 \%)$ and poor sleep quality by 88 women $(50.29 \%)$. The mean value of serum Magnesium level was $1.74 \pm 0.56 \mathrm{mg} / \mathrm{dl}$. Using Pearson's coefficient correlation, there was a positive correlation between sleep quality and serum magnesium level which was statistically significant.

Conclusion: Magnesium replacement therapy could be beneficial in improving the poor sleep quality in Premenstrual syndrome.

Keywords: Premenstrual syndrome, sleep quality, Magnesium.

\section{Introduction}

Premenstrual syndrome (PMS) is a cyclical disorder observed in late luteal phase presenting with behavioral changes that can affect interpersonal relationships and normal daily activity ${ }^{(1)}$. Clinical characteristics can

\author{
Corresponding Author: \\ Dr. R. Shanthini, M.D., \\ Associate Professor, Department of Physiology, Stanley \\ Medical College, Chennai \\ Mobile No.: 9159354414 \\ e-mail: shanphysio77@yahoo.in
}

be divided as physical, psychological and behavioral changes. PMS affects $90 \%$ of women in childbearing age. Sleep disturbances are also common. Women with PMS report sleep disturbances as increased sleep latency, number of awakenings and decreased sleep efficiency ${ }^{(1,2)}$. The most commonly used sleep questionnaire in assessing the subjective sleep quality is Pittsburgh Sleep Quality Index (PSQI). Progesterone, which is a hormone of luteal phase plays a key role in the pathogenesis of PMS. Apart from hormonal imbalances, micronutrients like magnesium,zinc and calcium are also implicated in the pathogenesis of $\mathrm{PMS}^{(3)}$.

Magnesium deficiencies causes depletion of brain dopamine without affecting brain serotonin and nor- 
epinephrine. It also increases threshold for stressful stimuli and in its deficiency aldosterone level is increased in response to environmental stimuli. Magnesium is an agonist of GABA ( $\gamma$-aminobutyric acid), the principle inhibitory neurotransmitter. At the same time, it is an antagonist of NMDA(N- methyl D- aspartate). NMDA and GABA play a key role in sleep regulation ${ }^{(3,4)}$.

There were limited studies using subjective method in order to detect sleep quality in PMS. In this study Pittsburgh Sleep Quality Index (PSQI) has been used to evaluate the subjective sleep quality. Score of more than 5 indicates poor sleep quality. While overnight Polysomnograph is a gold standard objective method, it has disadvantages like cost-effectiveness and equipment $^{(2)}$. Thus PSQI is practically good scale for estimating sleep disturbances. Here we also evaluate the correlation between the sleep quality and Serum Magnesium level. Sleep architecture, especially slow wave sleep, is closely associated with the glutamatergic and GABAergic system ${ }^{(5)}$

\section{Aim:}

- To diagnose the Premenstrual syndrome using Premenstrual Syndrome Scale (PMSS) in the women of age group 18 to 40 years

- To assess the subjective sleep quality in women with Premenstrual syndrome using Pittsburgh Sleep Quality Index (PSQI).

- To correlate subjective sleep quality with serum Magnesium level.

\section{Materials and Method}

A cross sectional study was conducted in 175 women with Premenstrual Syndrome who were recruited from Gynaecology department, RSRM, Stanley Medical College between July 2019 and September 2019.

\section{Inclusion Criteria:}

- Women with Premenstrual Syndrome

- $\quad$ Age group 18-40 years

- Women having regular menstrual cycles

\section{Exclusion Criteria:}

- Pregnancy or post partum period

- Chronic illness like Diabetes Mellitus, Hypertension, Chronic Kidney disease, Coronary Artery Disease

- Neurological illness
- Endocrine disorders

- Any gynaecological disorders

- Intake of OCP's and drug abuse

- Smoker, alcoholics and betel nut chewer

Data was collected after obtaining Ethical Committee clearance. The purpose of the study was explained to the subjects and a written informed consent was obtained. After Clinical Examination, Women diagnosed with Premenstrual Syndrome using "Premenstrual Syndrome Scale (PSS) were assessed for Subjective sleep quality using Pittsburgh Sleep Quality Index (PSQI).

Under sterile precautions $5 \mathrm{ml}$ of venous blood was collected and the serum was separated and stored in deep freezer. Serum Magnesium was estimated by Spectrophotometer atomic absorption technique

\section{Statistical Analysis:}

Data were collected and entered in MS Excel and analyzed using Epi Info software. The categorical variables were expressed in frequency and percentage and analyzed using Chi-square test. The continuous variables were expressed in mean and standard deviation. Pearson's coefficient correlation was used to determine the correlation between PMS and serum magnesium level. $p$ values $(p=<0.05)$ were considered as statistical significant

\section{Results}

Among the study population of 175 women with PMS, good sleep quality was reported by 88 women $(50.71 \%)$ and poor sleep quality by 87 women (49.29\%). The mean value of serum Magnesium level was $1.74 \pm 0.56 \mathrm{mg} / \mathrm{dl}$. There was a positive correlation between sleep quality and serum magnesium level which was stastically significant. The correlation coefficient $r=$ +0.2 which implies as the quality of sleep worsen serum Magnesium level.

Table 1: Demograpic and Clinical parameters of study population

\begin{tabular}{|l|c|}
\hline $\mathbf{n}=\mathbf{1 7 5}$ & Mean \pm SD \\
\hline Range & $26.94 \pm 6.32$ \\
\hline Age (18-40 yrs) & \\
\hline Marital Status & $110(43 \%)$ \\
\hline Married & $65(27 \%)$ \\
\hline Unmarried
\end{tabular}




\begin{tabular}{|l|c|}
\hline $\mathbf{n}=\mathbf{1 7 5}$ & Mean \pm SD \\
\hline PSQI & $1.85 \pm 0.43$ \\
\hline Subjective Sleep Quality (0-3) & $0.45 \pm 0.21$ \\
\hline Sleep Latency (0-3) & $0.54 \pm 0.23$ \\
\hline Sleep Duration (0-3) & $1.87 \pm 0.33$ \\
\hline Sleep Efficiency (0-3) & $1.21 \pm 0.46$ \\
\hline Sleep Disturbance (0-3) & 0 \\
\hline Use of Sleep Medication (0-3) & $1.60 \pm 0.34$ \\
\hline Daytime Dysfunction (0-3) & $88(51 \%)$ \\
\hline Global Score (0-21) & $87(49 \%)$ \\
\hline Good sleep Quality (0-5) & $1.86 \pm 0.42$ \\
\hline Poor Sleep Quality (6-21) & $1.75 \pm 0.25$ \\
\hline Serum Magnesium Level (1.7-2.4mg/dl) \\
\hline Good Sleepers & \\
\hline Poor Sleepers & \\
\hline
\end{tabular}

$\mathrm{P}<0.05$ Significant

\section{Discussion}

Sleep disturbance is one of the utmost concerns of women with PMS, which could even significantly affect their quality of life ${ }^{(6)}$. In accordance with Ozisik et al, there were significant difference in subjective sleep quality, sleep efficiency and daytime dysfunction with total PSQI score.We also see that there was a positive correlation between sleep quality and serum Magnesium level which was statistically significant ${ }^{(9)}$.

\section{Conclusion}

This study would serve as a wakeup call to give prime concern to the sleep related complaints to improve the quality of life in women with PMS. Dietary intake of micronutrients especially Magnesium can prevent or reduce the sleep related issues and can improve the quality of life.

Acknowledgement: We would like to express our sincere gratitude to all the people who helped us to carry our study. Authors acknowledge the immense help received from the scholars whose articles are cited and included in references of this manuscript. The authors are also grateful to authors/editors/publishers of all those articles,journals and books from where the literature for this article has been reviewed and discussed.

\section{Conflict of Interest if any: No}

Privacy/Confidentiality of the study subjects: Will be maintained.
Sponsor Details: Self

\section{Compensation: No}

Insurance: No

Glossary of Abbreviation:

OCP- Oral Contraceptive Pills

GABA- $\gamma$-aminobutyric acid

NMDA- N- methyl D- aspartate

PMS- Premenstrual Syndrome

PMSS- Premenstrual Syndrome Scale

PSQI -Pittsburgh Sleep Quality Index

\section{References}

1. OzisikKaraman HI, Tanriverdi G, Degirmenci Y. Subjective sleep quality in premenstrual syndrome. Gynecological Endocrinology. 2012 Aug 1;28(8):661-4.

2. Baker FC, Kahan TL, Trinder J, Colrain IM. Sleep quality and the sleep electroencephalogram in women with severe premenstrual syndrome. Sleep. 2007 Oct 1;30(10):1283-91.

3. Dickerson LM, Mazyck PJ, Hunter MH. Premenstrual syndrome. American family physician. 2003 Apr;67(8):1743-52.

4. Posaci C, Erten O, Üren A, Acar B. Plasma copper, zinc and magnesium levels in patients with premenstrual tension syndrome. Actaobstetricia et gynecologica Scandinavica. 1994 Jan 1; 73(6): 452-5.

5. Sherwood RA, Rocks BF, Stewart A, Saxton RS. Magnesium and thepremenstrual syndrome. Annals of clinical biochemistry. 1986 Nov;23(6):667-70.

6. Murck H. Magnesium and affective disorders. Nutritional neuroscience. 2002 Jan 1;5(6):375-89.

7. Lamarche LJ, Driver HS, Wiebe S, Crawford L, De Koninck JM. Nocturnal sleep, daytime sleepiness and napping among women with significant emotional/behavioral premenstrual symptoms. Journal of sleep research. 2007 Sep;16(3):262-8.

8. Direkvand-Moghadam A, Sayehmiri K, Delpisheh A, Kaikhavandi S. Epidemiology of Premenstrual Syndrome (PMS)-A systematic review and meta- 
analysis study. Journal of clinical and diagnostic research: JCDR. 2014 Feb;8(2):106.

9. Yonkers KA, O’Brien PS, Eriksson E. Premenstrual syndrome. The Lancet. 2008 Apr 5; 371(9619):1200-10.
10. Fathizadeh N, Ebrahimi E, Valiani M, Tavakoli N, Yar MH. Evaluating the effect of magnesium and magnesium plus vitamin B6 supplement on the severity of premenstrual syndrome. Iranian journal of nursing and midwifery research. 2010 Dec; 15 (Supp11):401. 